ARTICLE

https://doi.org/10.1038/s41467-019-09218-6

\title{
Ultra-strong long-chain polyamide elastomers with programmable supramolecular interactions and oriented crystalline microstructures
}

\author{
Lingzhi Song ${ }^{1}$, Tianyu Zhu ${ }^{2}$, Liang Yuan², Jiangjun Zhou' ${ }^{1}$ Yaqiong Zhang ${ }^{1}$, Zhongkai Wang (1) ${ }^{1} \&$ \\ Chuanbing Tang ${ }^{2}$
}

Polyamides are one of the most important polymers. Long-chain aliphatic polyamides could bridge the gap between traditional polyamides and polyethylenes. Here we report an approach to preparing sustainable ultra-strong elastomers from biomass-derived long-chain polyamides by thiol-ene addition copolymerization with diamide diene monomers. The pendant polar hydroxyl and non-polar butyrate groups between amides allow controlled programming of supramolecular hydrogen bonding and facile tuning of crystallization of polymer chains. The presence of thioether groups on the main chain can further induce metal-ligand coordination (cuprous-thioether). Unidirectional step-cycle tensile deformation has been applied to these polyamides and significantly enhances tensile strength to over 210 $\mathrm{MPa}$ while maintaining elasticity. Uniaxial deformation leads to a rearrangement and alignment of crystalline microstructures, which is responsible for the mechanical enhancement. These chromophore-free polyamides are observed with strong luminescence ascribed to the effect of aggregation-induced emission (AIE), originating from the formation of amide clusters with restricted molecular motions.

\footnotetext{
${ }^{1}$ Biomass Molecular Engineering Center, Anhui Agricultural University, Hefei, Anhui 230036, China. ${ }^{2}$ Department of Chemistry and Biochemistry, University of South Carolina, Columbia, SC 29208, USA. These authors contributed equally: Lingzhi Song, Tianyu Zhu. Correspondence and requests for materials should be addressed to Z.W. (email: wangzk6@ahau.edu.cn) or to C.T. (email: tang4@sc.edu)
} 
ong-chain aliphatic polyesters and polyamides possess some of unique compositions and properties, given that they bridge the gap between conventional polyethylene and short-chain condensation polymers (or polycondensates) ${ }^{1-4}$. While the long aliphatic chain promotes crystallization via van der Waals interactions, the presence of functional groups (e.g., esters and amides) powers this class of polymers for applications that polyethylene cannot enable such as degradability and compostability 5,6 . Coupling with possible feedstocks from renewable natural resources, these type of polymers would hold enormous potential in pursuing sustainability toward green bioplastics $^{7-17}$. Thus, there is an indigenous incentive to design viable routes to access long-chain monomers and their corresponding polymers. One of the primary approaches is to prepare linear $\alpha$, $\omega$-difunctional monomers, including selective terminal functionalization of fatty acids, which are corroborated by recent advances in catalytic conversions of plant oils ${ }^{18-20}$. However, many of these polymers require tedious synthetic processes and exhibit inferior physiochemical properties compared with either polyethylene or traditional condensation polymers. Most of them are reported as thermoplastics with just a few on elastomers with mediocre mechanical properties.

As shown in Fig. 1, we conceptualized castor oil-derived amide diene monomers. The key advantage of our design is the introduction of hydroxyl groups in the main chain that could be further used to install functional groups as side chains, which has been rarely reported with other polyamide systems. Two monomers with hydroxyl or butyrate pendant groups were first synthesized. Functional polyamides were then prepared via thiol-ene addition polymerization. Copolymerization of these monomers would allow precise tuning of crystallization properties of resultant polyamides. The existence of butyrate pendant groups may restrain the formation of a highly crystalline structure, but facilitate an elastic amorphous matrix. Nanocrystals from the packing of linear alkyl chains and supramolecular hydrogen bonding from amide/hydroxyl side groups contribute to high mechanical strength. Moreover, it is worth noting that the ultrahigh mechanical strength could be achieved via the formation of oriented microstructures ${ }^{21-23}$. Unidirectional step-cycle tensile deformation was therefore performed on these functional polyamides to induce alignment of microstructures. Ultra-strong elastomers (uEs) with oriented nanocrystals dispersed in the amorphous matrix were obtained.

On a different perspective, a variety of small molecules and macromolecules exhibit enhanced luminescence rather than quenching at the solid state, a phenomenon coined as aggregation-induced emission (AIE) by Tang et al. ${ }^{24-26}$. Very interestingly, many chromophore-free synthetic polymers (e.g., anhydride, amide-rich polymers) ${ }^{27-29}$ and natural biopolymers (e.g., cellulose, starch, peptide $)^{27}$ also show unusual emission at the aggregation state. Given the rich content of amide groups, we hypothesize that our polyamides could have similar amide cluster-induced luminescence, which could bring additional benefits of this class of materials.

We report herein the design of a class of biobased long-chain aliphatic polyamides that combine van der Waals interactions and supramolecular hydrogen bonding. The macromolecular compositions facilitate the formation of crystalline nanostructures dispersed in an amorphous matrix. With the controlled programming of supramolecular interactions, it would allow the facile tuning of mechanical strength. Further unidirectional stretching of these polyamide films induces the orientation of a<smiles>C=CCCCCCCCCC(=O)OC</smiles>

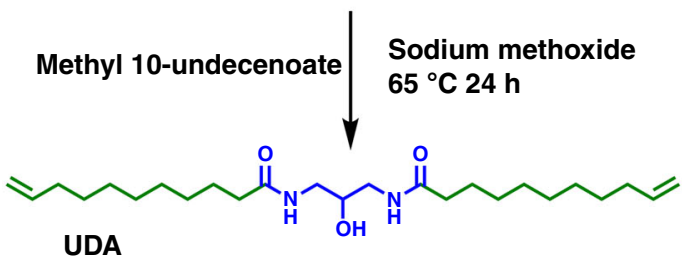

b<smiles>[2H]OC(CNC(=O)CCCCCCCC=C)CNC(=O)CCCCCCCCC=C</smiles>

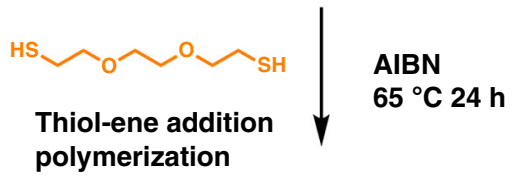<smiles>C=CCCCCCCCCC(=O)NCC(CNC(=O)CCCCCCCCC=C)OC(=O)CCC</smiles>

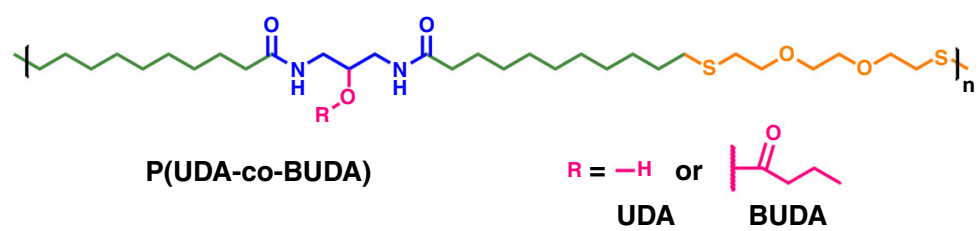

C

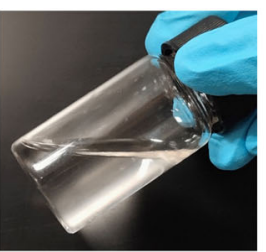

Methyl 10-undecenoate

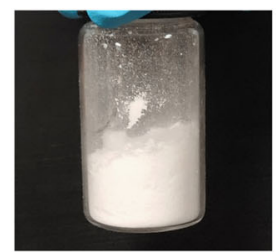

UDA

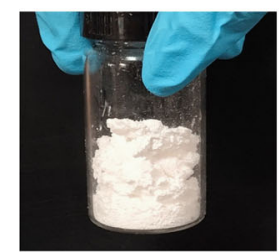

BUDA

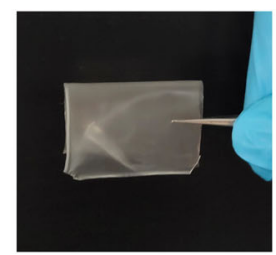

PBUDA film

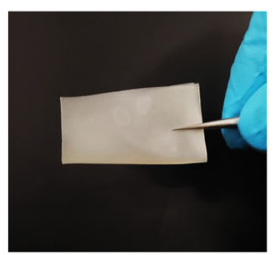

$\mathrm{P}($ UDA-co-BUDA) film

Fig. 1 Design of monomers and functional polyamides. a Synthesis of amide diene monomers from a castor oil derivative; $\mathbf{b}$ synthesis of polyamides by thiol-ene addition polymerization; c photos represent methyl 10-undecenoate, amide diene monomers (UDA: N,N'-(2-hydroxypropane-1,3-diyl)bis(undec10-enamide), BUDA: 1,3-di(undec-10-enamido)propan-2-yl butyrate), PBUDA and P(UDA-co-BUDA) films 
ordered crystalline domains and thus results in ultra-strong elastic materials with an increase of tensile strength by nearly one order of magnitude. This kind of biobased polyamides is the strongest elastomers reported among long-chain aliphatic polycondensates.

\section{Results}

Synthesis of long-chain polyamides. Recently, several approaches have been developed to prepare polyamides with functional side groups ${ }^{30-33}$. However, the synthesis of monomers was mostly involved with tedious work-up and relatively low yields. In this work, we developed efficient synthesis of diene monomers that bear two secondary amide bonds in the main chain attached with pendant side groups. As shown in Fig. 1a, an $\alpha, \omega-$ diene amide monomer with hydroxyl side group ( $N, N^{\prime}$ (2-hydroxypropane-1,3-diyl)bis(undec-10-enamide), UDA) was first prepared via amidation of methyl 10-undecenoate with 1,3diamino-2-propanol ${ }^{34-36}$. The reaction conversion into UDA monomer during this step is quantitative $(\sim 100 \%)$. The hydroxyl group in UDA was then reacted with butyric anhydride to introduce a larger pendant group that could later inhibit crystallization of its polymer. The resultant monomer 1,3-di (undec-10-enamido)propan-2-yl butyrate is labeled as BUDA. The successful synthesis of UDA and BUDA was confirmed by Fourier-transform infrared spectroscopy (FT-IR), proton nuclear magnetic resonance $\left({ }^{1} \mathrm{H}\right.$ NMR), and carbon-13 nuclear magnetic resonance spectroscopy (Supplementary Fig. 1). Thiol-ene addition polymerization of both diene monomers was performed to afford polyamides (Fig. 1b, labeled as PUDA and PBUDA, respectively). The existence of hydrogen bonded amide groups along with linear alkyl chains would result in highly crystalline polymers for PUDA. Indeed, PUDA homopolymer (P7) is a highly crystalline and brittle material (Supplementary Fig. 2a). On the other hand, the presence of pendant groups could interrupt the formation of crystalline domains in PBUDA (P0, transparent in Fig. 1c). Thus, we predict that a copolymer of both monomers (P(UDA-co-PBUDA) (opaque in Fig. 1c) could have the existence of a two-phase morphology with nanocrystalline domains dispersed in an amorphous matrix. The precise control of feed ratios of co-monomers would allow the facile tuning of crystallinity with the aid of programmable supramolecular hydrogen bonding.

To understand the effect of monomeric compositions on microstructures and thermomechanical properties of functional polyamides, we prepared a series of P(UDA-co-BUDA) copolymers with the fraction of UDA varying from 10 to $80 \mathrm{~mol} \%$ (polyamides P1-P6 in Supplementary Table 1). The formation of these polyamide copolymers was confirmed by ${ }^{1} \mathrm{H}$ NMR (Supplementary Fig. 3), gel permeation chromatography (Supplementary Fig. 4), and thermogravimetric analysis (Supplementary Fig. 5). All these polyamides have relatively high molecular weight $\left(>20,000 \mathrm{~g} \mathrm{~mol}^{-1}\right)$ and good thermal stability.

Differential scanning calorimetry (DSC) was performed to estimate the microstructure of polymers. Figure 2a shows heat flow curves of DSC for P0-P7. All copolymers exhibited glass transition temperature $\left(T_{\mathrm{g}}\right)$ far below room temperature $(-29.4$ to $-21.6{ }^{\circ} \mathrm{C}$ ). Moreover, DSC curves show distinct melting and crystallization processes for both PUDA homopolymer (P7) and copolymers (P1-P6). The melting temperature $\left(T_{\mathrm{m}}\right)$ of $\mathrm{P} 1-\mathrm{P} 7$ increased with the increase of UDA content. PUDA homopolymer (P7) has the highest $T_{\mathrm{m}}$ at $\sim 122.3{ }^{\circ} \mathrm{C}$ and an enthalpy of fusion $\left(\Delta H_{\mathrm{m}}\right)$ of $595.5 \mathrm{Jg}^{-1}$, while PBUDA homopolymer (P0) was not observed with a melting point. The DSC heating curves for polymers with high UDA content (P5-P7) show two distinct melting peaks, which can be interpreted by a coexistence of the $\gamma$-phase and $\alpha^{\prime}$-phase crystals at elevated temperature ${ }^{37,38}$. These results suggest the incorporation of UDA with the hydroxyl side group facilitates the formation of crystalline domains: the higher the UDA content, the higher melting temperature.

Microstructures and supramolecular hydrogen bonding. Wideangle X-ray diffraction (WAXD) was further used to probe crystallization behaviors of homopolymers of UDA and BUDA, as well as their copolymers (Fig. 2b). Except PBUDA homopolymers, all other polymers were observed with a broad baseline and sharp peaks with varied intensity, indicating the coexistence of both crystalline and amorphous microstructures. The crystalline and amorphous peaks were deconvoluted via peak fitting (Supplementary Fig. 6). The crystalline peak at $2 \theta$ around $21.2^{\circ}$ corresponds to the $\gamma$-crystalline form as reported for polyamides ${ }^{39}$. The degree of crystallinity $\left(X_{c}\right)$ was calculated based on the fraction of areas under crystalline peaks over the total areas under both crystalline and amorphous regions. Supplementary Table 2 summarizes the peak positions and $X_{c}$ for $\mathrm{P} 0-\mathrm{P}^{40} . X_{\mathrm{c}}$ increases with the increase of UDA content in the copolymers, in good agreement with the DSC data. The size of crystalline domains $(t)$ can be estimated from the Scherrer's formula: $t=\lambda / B \cos \theta$, where $\lambda$ is the wavelength of $\mathrm{X}$-ray, $B$ is the full width at half-maximum of diffraction peaks, and $\theta$ is the diffraction angle. As a result, the size of crystalline domains is only ca. $6.7-8.5 \mathrm{~nm}$ for the copolymers. Although well-aligned UDA segments induce the growth of crystallites, the pendant butyrate groups hinder the alignment polyethylene-like chains at the backbone and further depress the overall strength of intermolecular van der Waals interactions. Supplementary Figure 7 shows a polarized optical micrograph (POM) of a representative copolymer P4, the nearly dark image also indicated that the crystals are too small to be visualized by POM.

It was conceptualized that supramolecular hydrogen bonding should play a critical role in the crystallization of polyamides. As hydrogen bonding is temperature-sensitive, these copolymers were characterized by variable temperature FT-IR spectroscopy. When $\mathrm{C}=\mathrm{O}$ groups of amides form hydrogen bonds, their infrared absorption peak may shift. Figure $2 \mathrm{c}$ shows FT-IR spectra of P0-P7 in the $1500-1700 \mathrm{~cm}^{-1}$ region. The peaks near $1620-1680 \mathrm{~cm}^{-1}$ are assigned to the stretching of carbonyl groups. This region of carbonyl stretching contains three distinct contributions: free carbonyl groups at $1678 \mathrm{~cm}^{-1}$, disordered hydrogen bonded carbonyl groups at $1646 \mathrm{~cm}^{-1}$, and ordered hydrogen bonded carbonyl groups at $1626 \mathrm{~cm}^{-1}$, the latter two of which indicate the formation of hydrogen bonding in polyamides ${ }^{41}$. Figure $2 \mathrm{~d}$ shows the changes of peak intensities of free/ disordered hydrogen bonded/ordered hydrogen bonded carbonyl groups as a function of UDA content for polymers $\mathrm{P} 0-\mathrm{P} 7$ at room temperature. It can be seen that the peak intensity of ordered hydrogen bonded groups greatly increased with the increase of UDA content, demonstrating that the presence of more UDA facilitated the formation of hydrogen bonding. Figure 2e shows variable temperature FT-IR spectra of a representative copolymer P6 in the $1500-1700 \mathrm{~cm}^{-1}$ region, which indicates association/ dissociation of hydrogen bonds under different temperature. With the increase of temperature, the absorption peaks at 1646 and $1626 \mathrm{~cm}^{-1}$ decreased, and a broad peak near $1678 \mathrm{~cm}^{-1}$ gradually increased, indicating the weakening and dissociation of hydrogen bonds. Figure $2 \mathrm{f}$ shows the changes of peak intensity of free/disordered hydrogen bonded/ordered hydrogen bonded $\mathrm{C}=\mathrm{O}$ for $\mathrm{P} 6$ as a function of temperature. There is a transition occurred from $80^{\circ} \mathrm{C}$ to $120^{\circ} \mathrm{C}$, which is in excellent agreement with the melting results by DSC. This suggested that the ordered hydrogen bonds exist mostly within the crystalline region. Based on these observations, molecular models for crystalline and 

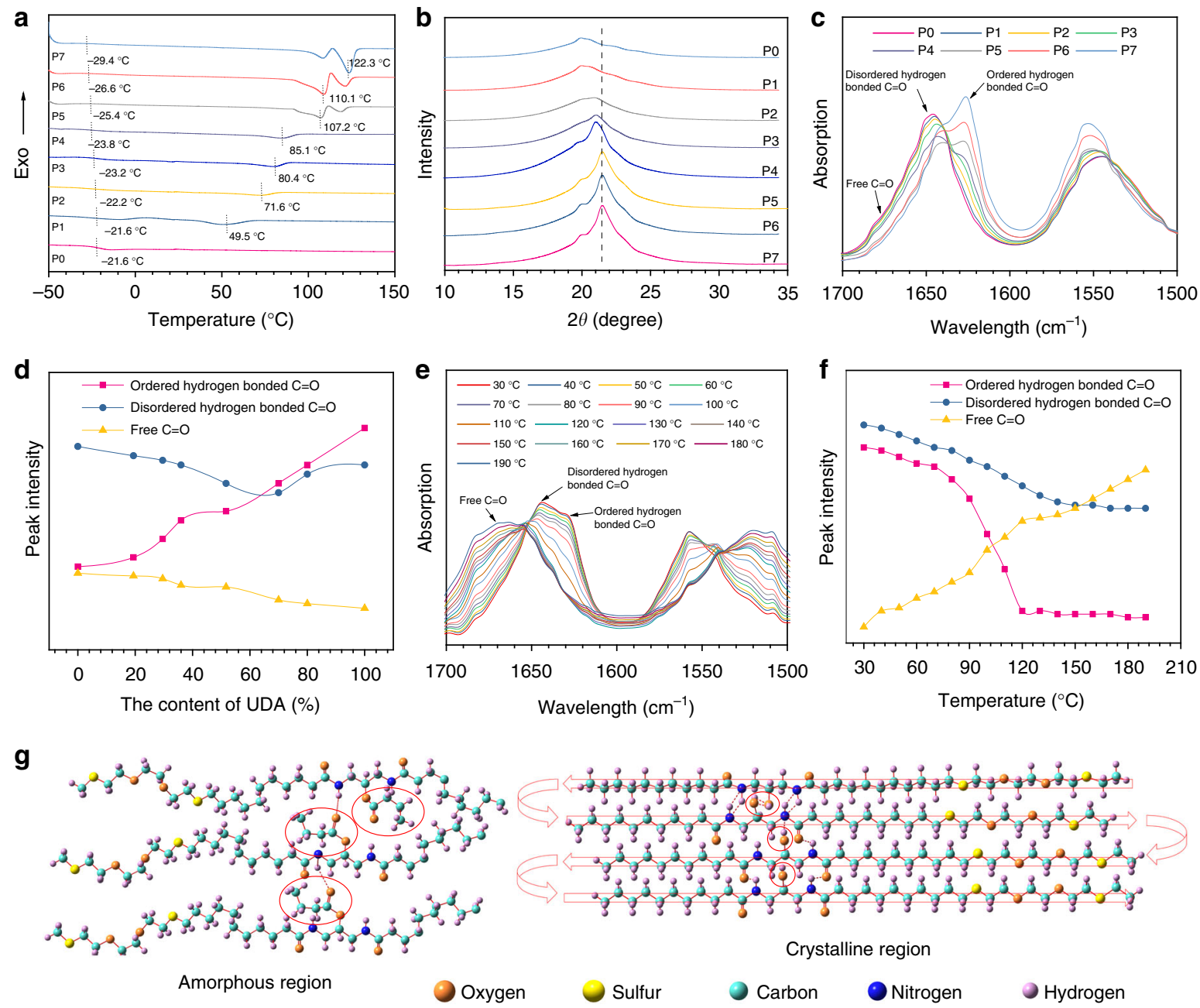

Fig. 2 Characterization of polyamides PO-P7. a Differential scanning calorimetry (DSC) heating curves; b one-dimensional (1D) wide-angle X-ray diffraction (WAXD) profiles; c Fourier-transform infrared spectroscopy (FT-IR) spectra in the $1500-1700 \mathrm{~cm}^{-1}$ region for PO-P7; d the change of FT-IR peak intensity of free/disordered/ordered hydrogen bonded $\mathrm{C}=\mathrm{O}$ for $\mathrm{PO}-\mathrm{P} 7$ as a function of UDA content; e variable temperature FT-IR spectra of P6 in the $1500-1700$ $\mathrm{cm}^{-1}$ region; $\mathbf{f}$ the change of FT-IR peak intensity of free/disordered/ordered hydrogen bonded $\mathrm{C}=\mathrm{O}$ for $\mathrm{P} 6$ as a function of temperature; $\mathbf{g}$ proposed molecular models for crystalline domain and amorphous region

amorphous phases were proposed in Fig. $2 \mathrm{~g}$. The large butyrate side groups of BUDA disrupt chain regularity and prefer to stay in the amorphous region, while hydroxyl groups facilitate crystallization. Overall, it can be concluded that in addition to intermolecular van der Waals interactions from linear alkyl chains, the formation of semicrystalline microstructures was largely facilitated by inter/intra-molecular hydrogen bonding.

The dispersion of crystalline microstructures in an amorphous matrix is crucial to possess outstanding mechanical properties for many of polyamide systems ${ }^{42-44}$. For the copolymers of UDA and BUDA, an amorphous matrix envelopes nanocrystalline domains, where there are rich inter/intra-molecular hydrogen bonds. The mechanical properties of copolymers with various UDA contents were measured via monotonic tensile deformation (Fig. 3a, Supplementary Fig. 8, and Supplementary Table 3). Compared to the non-crystalline homopolymer PBUDA, copolymer P4 has tensile strength and Young's modulus at $18.4 \pm 2.1$ and $149.6 \pm 3.1 \mathrm{MPa}$, an increase of $429.6 \%$ and $521.4 \%$, respectively. Moreover, with the increase of UDA content, the toughness increased from $5.2(\mathrm{P} 0)$ to $65.0 \mathrm{MJ} \mathrm{m}^{-3}$ (P4), an increase over 12.5-folds, demonstrating that the existence of nanocrystalline domains greatly enhances toughness. Copolymers P1-P3 were also observed with similar trends at different levels of increase in mechanical properties (Fig. 3a). Copolymers P5-P6 with higher contents of UDA (60\% and $80 \%$ respectively) were not subject to such comparisons, as they are quite brittle.

Preparation of uEs. It is expected that the as-prepared film samples of copolymers by solution casting do not possess highly oriented crystalline microstructures. Consecutive cyclic tensile deformation was applied to copolymers P1-P4 to induce the alignment of microstructures ${ }^{45,46}$. After deformation, these samples were labeled as $\mathrm{uE} 1-\mathrm{uE} 4$ (Fig. 3 and Supplementary Figs. 9-11). The stress-strain curves during the first and second step-cycle tensile deformation of $\mathrm{P} 4$ are shown in Fig. $3 \mathrm{~b}$ and c. The large hysteresis during each cycle is in agreement with the Mullin effect ${ }^{47}$. After the step-cycle tensile deformation, these copolymers were transformed into ultra-strong elastomers (uE1-uE4). For example, the stress at break of uE4 is $126.3 \mathrm{MPa}$, more than seven times of as-prepared P4 (Fig. 3e). Though the elongation at break of $\mathrm{uE} 4$ reduced to $30 \%$, its elastic recovery is strikingly high at $94.5 \%$ (Fig. 3d). Similarly, the high elastic recovery was also observed for $\mathrm{uE} 1, \mathrm{uE} 2$, and $\mathrm{uE} 3$ at $96.9 \%$, $96.7 \%$, and $95 \%$ respectively (Supplementary Fig. 12). Together, these elastomers combined ultrahigh tensile strength and 

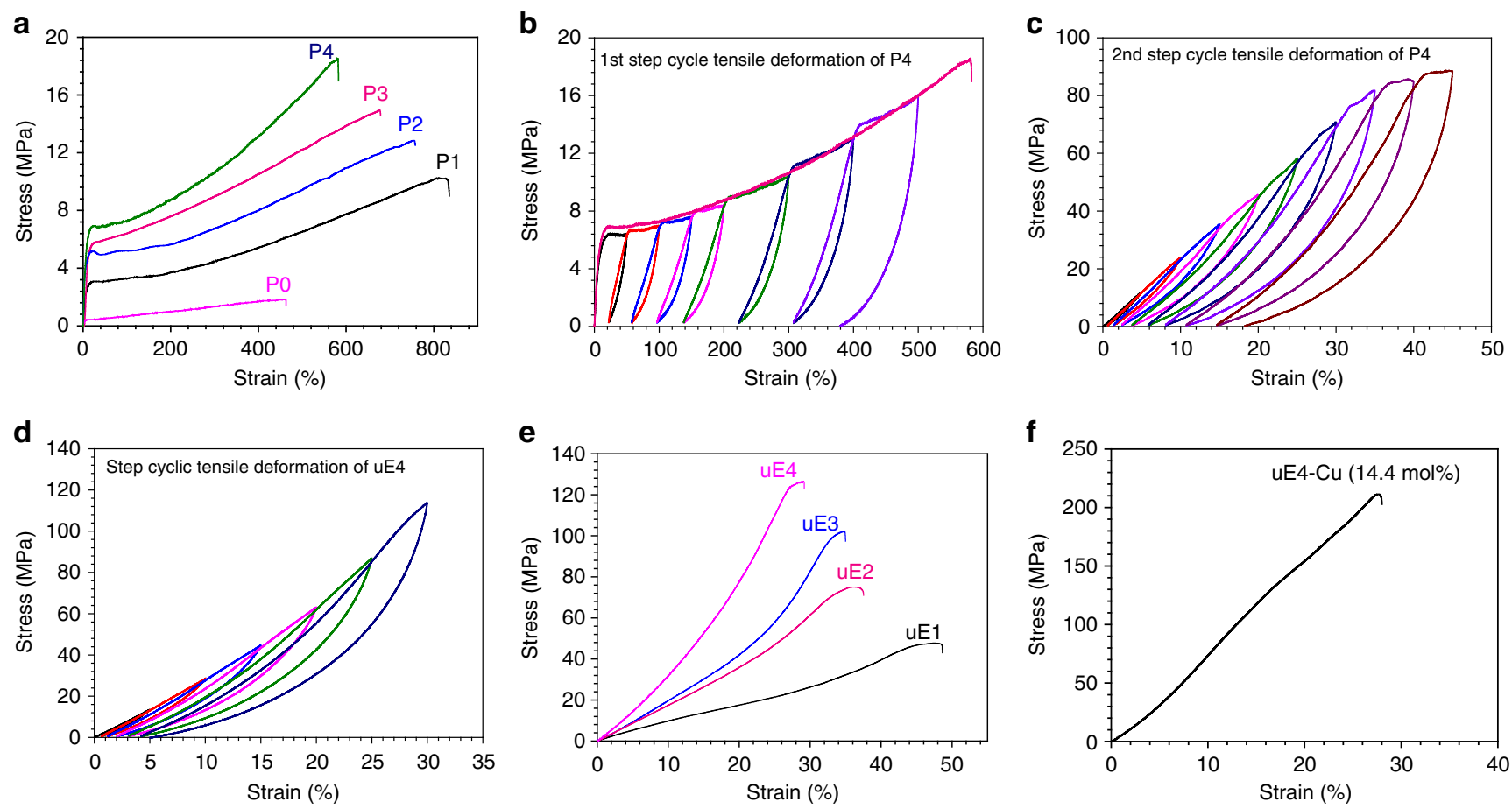

Fig. 3 Step-cycle tensile deformation as a processing method to improve mechanical properties. a Monotonic stress-strain curves of P0-P4; $\mathbf{b}$ first and c second step-cycle tensile deformation of P4; $\mathbf{d}$ monotonic stress-strain curves of ultra-strong elastomer 4 (uE4) during step-cycle tensile deformation; e monotonic stress-monotonic strain curves of uE1-uE4; f monotonic stress-strain curve of uE4-Cu (14.4 mol\%)

excellent elasticity. The mechanical properties of $\mathrm{uE} 1-\mathrm{uE} 4$ are summarized in Supplementary Table 4 . The mechanical properties of uEs can be precisely tuned by controlling the content of UDA. Supplementary Figure 13 shows that a uE4 fiber with a diameter of $\sim 135 \mu \mathrm{m}$ can easily hold a weight of $200 \mathrm{~g}$, implying the exceptional toughness.

To demonstrate that there is much room for further improving mechanical strength of these polyamides, we carried out a preliminary study by introducing metal-ligand coordination. Cuprous-thioether coordination has been widely observed in biological systems and recently used in synthetic polymers ${ }^{48-51}$. Our polyamides contain a reasonable fraction of thioether groups along the backbone. Thus, $\mathrm{CuBr}$ was used to induce cuprous-thioether coordination to strengthen the mechanical properties (Supplementary Fig. 22). After similar tensile deformation processing, as shown in Fig. 3f and Supplementary Figs. 1415 , polyamides with $14.4 \mathrm{~mol} \%$ cuprous ions (to the sulfur atoms, labeled as uE4-Cu) exhibit stress at break at $211.2 \mathrm{MPa}$, more than $65 \%$ increase over $\mathrm{uE} 4$, while maintaining similar elongation at break $(27.9 \%)$. This substantial enhancement is a strong indication of the robustness of current structures of polyamide systems.

To place our work in context, we have collected a variety of longchain polyamides and polyesters reported in literature and compared their tensile strength (Supplementary Table 5). It is quite evident that the polyamides we prepared possess the highest tensile strength, even much higher than long-chain nylons (Nylon 12). While it may not be fair to compare, our polymers also have better tensile strength than short-chain nylons (e.g., polyamide 6.6).

To understand the substantial difference of mechanical properties between as-prepared $\mathrm{P} 1-\mathrm{P} 4$ and tensile-deformed $\mathrm{uE1}-\mathrm{uE4}$, WAXD measurement was used to reveal how the microstructures could be rearranged by the cyclic tensile deformation. As shown in Fig. 4a, 2D WAXD pattern of P4 is nearly isotropic. After cyclic tensile processing, an anisotropic 2D WAXD pattern is clearly formed for $\mathrm{uE} 4$, the arrow represents the stretching direction (Fig. 4b). The scattering intensity was found to converge on the meridian, which indicates that crystalline domains are oriented along the tensile direction. The orientation of crystal phase is also verified by the azimuthal angle at $2 \theta=21^{\circ}$ (Fig. 4c). According to the WAXD analysis (Supplementary Figs. 16-18), we proposed a model on microstructure rearrangement of P(UDA-co-BUDA) copolymers during cyclic tensile deformation (Fig. 4d).

AIE of polyamides. Surprisingly, both as-prepared and unidirectionally stretched polyamides exhibited strong luminescence. As shown in Fig. 5a and b, as-prepared P4 emits strong blue photoluminescence. The corresponding fluorescent spectrum shows an emission peak at $418 \mathrm{~nm}$, while the UV-vis absorption peak is at $210 \mathrm{~nm}$ (Fig. 5c and Supplementary Fig. 19). Figure $5 \mathrm{~d}-\mathrm{f}$ show fluorescence images of stretched $\mathrm{uE} 4$ fibers observed under microscope with a diode laser, which was excited with 340-380, 460-495, and 530-550 nm, respectively. Moreover, Fig. $5 \mathrm{~g}$ indicates that the emission color of $\mathrm{P} 4$ film did not show obvious difference during stretching, which was further corroborated by fluorescent spectra of P1-P4 and uE1-uE4 (Supplementary Fig. 20). Traditional chromophore-containing fluorescent polymers do not emit strong fluorescence in concentrated solutions or solid states due to the aggregation-caused quenching 24,52 . In the current case, the as-prepared polyamides without any conventional chromophores exhibit strong emission at the solid state. We believe that these polyamides have the properties of AIE (Supplementary Fig. 21), which was first discovered with chromophore-containing molecules by Tang and coworkers in 200125. Recently, synthetic and natural chromophore-free polymers have been reported with AIE characteristics, as a result of the formation of polar group clusters due to restriction of molecular motions ${ }^{27,53}$. A few research groups have reported polyamides with photoluminescence ${ }^{29,54-56}$. It is 

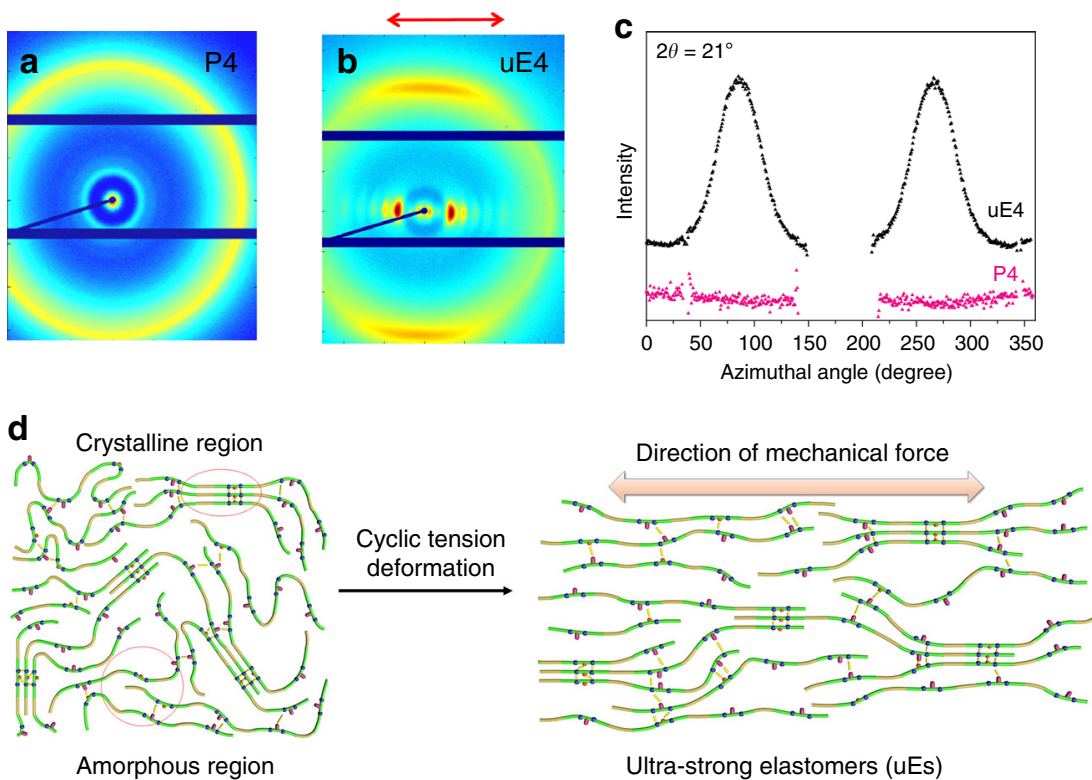

Fig. 4 Microstructure analysis of as-prepared and tensile-deformed copolymers. a, b Two-dimensional (2D) wide-angle X-ray diffraction (WAXD) patterns of P4 and ultra-strong elastomer 4 ( $\mathrm{UE} 4$ ); c WAXD azimuthal intensity profiles for P4 and uE4 at $2 \theta=21^{\circ}$; $\mathbf{d}$ a proposed model to illustrate the microstructure rearrangement of $\mathrm{P}(\mathrm{UDA}-\mathrm{co}-\mathrm{BUDA})$ copolymers during cyclic tensile deformation

generally believed that the strong hydrogen bonding induces the formation of local clusters of amides that facilitate fluorescence. We hypothesized that our polymers with rich amide and hydroxyl groups enable through-space conjugation via $n-\pi^{*}$ or $\pi-\pi^{\star}$ transitions ${ }^{52}$. Thus, the as-prepared polyamides and elastomers could generate strong fluorescence in the solid state (Fig. 5h), which could be beneficial for the extension of these elastomers from bioplastics to other areas such as biomedical applications that are worthy for exploration in the future.

In summary, biobased $\alpha$, $\omega$-diene amide monomers were designed with pendant polar hydroxyl or non-polar butyrate groups. Compared with traditional polyamides, these long-chain polyamides prepared by thiol-ene addition polymerization have controlled programmable supramolecular hydrogen bonding and tunable crystallinity with mechanical properties facilely adjusted by changing co-monomer compositions. By unidirectional stepcycle tensile deformation, ultra-strong elastomers were obtained without the addition of any fillers. It is the formation of highly aligned crystalline microstructures responsible for the unique mechanical properties of elastomers. Moreover, the clustered amide groups with molecular motions restricted in the aggregate state enable these polyamides with luminescence, a phenomenon of AIE. This study provides an approach to pursuing biobased polymers derived from renewable natural products, which combine ultrahigh mechanical strength, excellent elasticity, and strong photoluminescence.

\footnotetext{
Methods

Synthesis of UDA. We revised a procedure reported earlier ${ }^{11}$. UDA was prepared via amidation using 1,3-diamino-propanol (Fig. 1a). A typical procedure is given as follows: 1,3-diamino-propanol (10 mmol, $0.93 \mathrm{~g})$ and methyl 10-undecenoate $(20 \mathrm{mmol}, 4.13 \mathrm{~g})$ were charged into a $25 \mathrm{~mL}$ round-bottom flask. After purging nitrogen at $100^{\circ} \mathrm{C}$ for $30 \mathrm{~min}$, the reaction mixture was cooled to $65^{\circ} \mathrm{C}$, and $0.1 \mathrm{~mL}$ of sodium methoxide $(0.5 \mathrm{mmol}, 27 \mathrm{mg}$ in $30 \mathrm{wt} \%$ methanol $)$ and $3 \mathrm{~mL}$ of anhydrous tetrahydrofuran (THF) were added. The reaction ran for $12 \mathrm{~h}$. Pure and white UDA powder ( $3.0 \mathrm{~g}, 63 \%$ yield) was obtained via recrystallization from methanol twice and dried under vacuum.
}

Synthesis of BUDA. Monomer (BUDA) was prepared by esterification between UDA with butyric anhydride (Fig. 1a). A typical procedure is described as follows: UDA $(8.0 \mathrm{mmol}, 3.38 \mathrm{~g})$, butyric anhydride $(8.4 \mathrm{mmol}, 1.33 \mathrm{~g})$, 4-dimethylaminopyridine
$(0.24 \mathrm{mmol}, 30.0 \mathrm{mg})$, and THF $(4 \mathrm{~mL})$ were added into a $25 \mathrm{~mL}$ round-bottom flask After reacting at $60^{\circ} \mathrm{C}$ for $24 \mathrm{~h}$, deionized water $(1 \mathrm{~mL})$ and THF $(4 \mathrm{~mL})$ were injected to the mixture to quench the unreacted anhydride. The reaction solution was then poured into dichloromethane and washed with aqueous solutions of $\mathrm{NaHCO}_{3}$ and $\mathrm{NaCl}$. White BUDA solid ( $2.7 \mathrm{~g}, 69 \%$ yield) was obtained by drying the organic phase and evaporating the solvent.

Preparation of polyamides. Polyamides were prepared via thiol-ene addition polymerization of UDA and BUDA with dithiol. Azobisisobutyronitrile (AIBN) was used as a radical initiator. A typical procedure is described as follows (using BUDA as an example): BUDA (2.0 mmol, $0.99 \mathrm{~g}), 3,6$-dioxa-1,8-octanedithiol $(2.0 \mathrm{mmol}, 0.38 \mathrm{~g})$, AIBN $(0.06 \mathrm{mmol}, 10 \mathrm{mg})$, and THF $(2 \mathrm{~mL})$ were charged into a $10 \mathrm{~mL}$ flask, purged with nitrogen for $10 \mathrm{~min}$, and reacted at $70^{\circ} \mathrm{C}$ for $12 \mathrm{~h}$. The reaction mixture was diluted with THF and precipitated in methanol for several times. The precipitates were dried under vacuum at $40^{\circ} \mathrm{C}$ to obtain homopolymer of PBUDA (P0) (64\% yield). All other polyamides were prepared in a similar procedure.

Preparation of polyamide films. P0-P4 films were prepared by solution casting. A typical procedure is described as follows: polyamides $(1.0 \mathrm{~g})$ were dissolved in THF $(7 \mathrm{~mL})$ and poured into a Teflon mold. Films were dried at room temperature for 2 days, and then under vacuum at $40^{\circ} \mathrm{C}$ for $24 \mathrm{~h}$. Fibers of uE4 were prepared via a wire-drawing process. Typically, $\mathrm{P} 4(1.0 \mathrm{~g})$ was introduced into a flask and heated to $120^{\circ} \mathrm{C}$. After melting, fibers were drawn from the melt using a tweezer.

Preparation of metal-ligand coordination polyamide films. A typical procedure was described as follows: $\mathrm{P} 4(700 \mathrm{mg}), \mathrm{CuBr}(6 \mathrm{wt} \%, 43.2 \mathrm{mg})$, and dimethylformamide $(6 \mathrm{~mL})$ were added into a $25 \mathrm{~mL}$ round-bottom flask. The mixture was purged with nitrogen for $15 \mathrm{~min}$ and heated at $65^{\circ} \mathrm{C}$ for $8 \mathrm{~h}$, followed by rotary evaporation and vacuum dry at $80^{\circ} \mathrm{C}$ for $72 \mathrm{~h}$. Films were obtained by hot press at $120^{\circ} \mathrm{C}$.

Preparation of uEs. Polymers P0-P4 were performed on a SUNS UTM2502 instrument with a $100 \mathrm{~N}$ load cell. The crosshead speed was set at $10 \mathrm{~mm} \mathrm{~min}^{-1}$. The maximum strain was stretched to $100 \%, 200 \%, 300 \%$, up to $600 \%$ during the first step-cycle tensile deformation. The maximum strain during the second stepcycle tensile deformation was gradually increased from 5 to $40 \%$. The elastic recovery of uEs was calculated using the equation: Elastic recovery $=\left(\varepsilon_{\max }-\varepsilon_{0}\right) /$ $\varepsilon_{\max }$, where $\varepsilon_{\max }$ and $\varepsilon_{0}$, respectively, represent the maximum strain and the strain at $0 \mathrm{MPa}$ in each cycle. The preparation of metal-ligand coordination-based ultrastrong elastomers $(\mathrm{uE}-\mathrm{Cu})$ was carried out using the similar procedure to the above.

\section{Data availability}

All the data supporting the findings of this study are available within the article and its Supplementary Information file or from the corresponding authors upon request. 

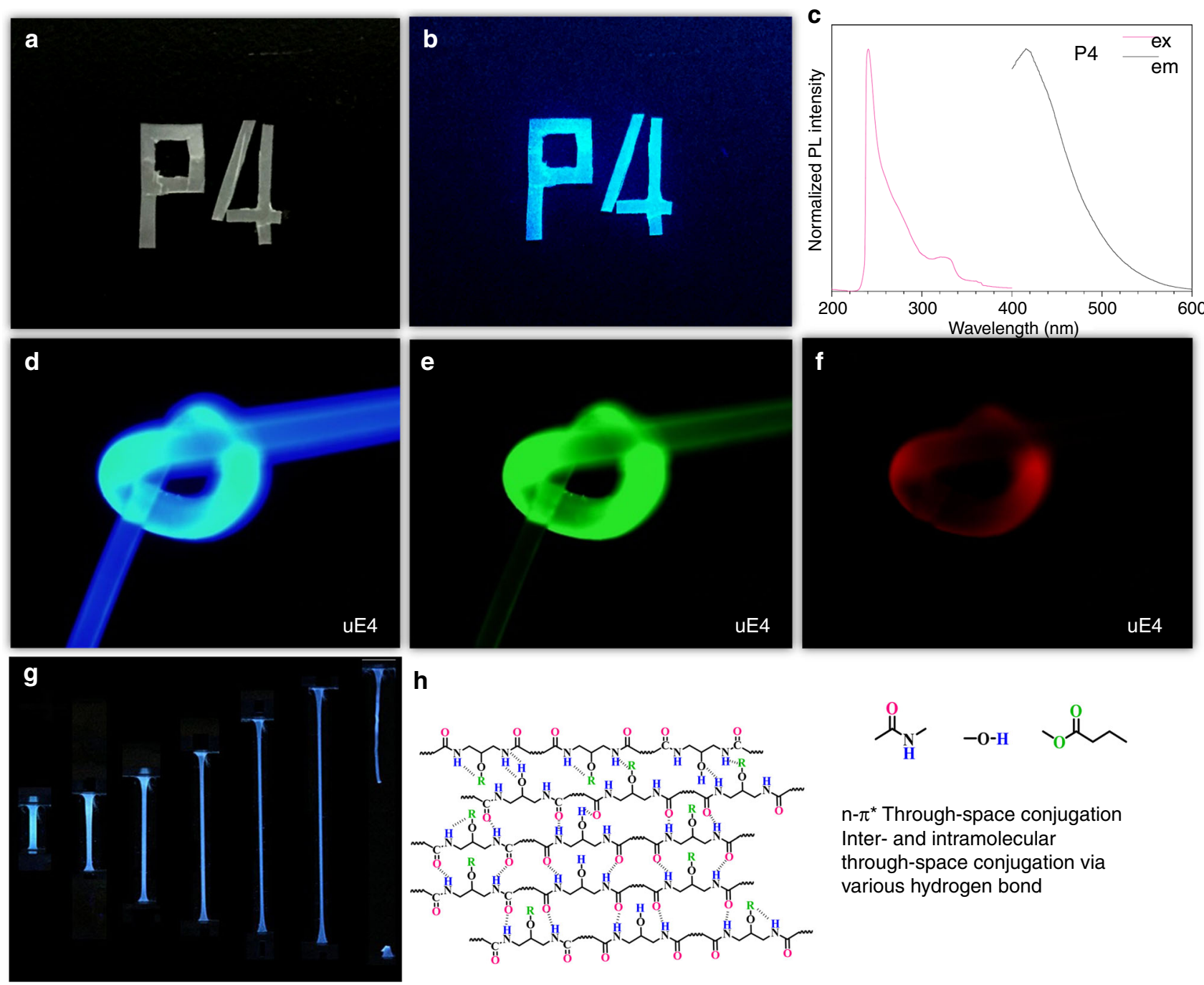

h

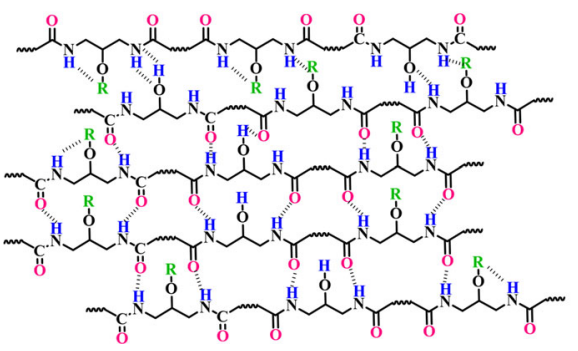<smiles>CCCC(=O)OC</smiles>

$\mathrm{n}-\pi^{*}$ Through-space conjugation

Inter- and intramolecular

through-space conjugation via

various hydrogen bond

Fig. 5 Aggregation-induced emission (AIE) properties of as-prepared polyamides and ultra-strong elastomers (uEs). a Photo of P4 under visible light; b photo of P4 under $365 \mathrm{~nm}$ light, emitting strong blue fluorescent; c ultraviolet-visible (UV-vis) absorption (red line) and fluorescent emission (black line) spectra of P4; $\mathbf{d}-\mathbf{f}$ fluorescence images of uE4 fibers observed under a microscope with a diode laser under varied excited wavelengths (340-390, 460-495, and 530-550nm); $\mathbf{g}$ photos of P4 during stretching excited by $365 \mathrm{~nm}$ UV lamp; $\boldsymbol{h}$ schematic illustration on the luminescent mechanism of polyamides

Received: 28 October 2018 Accepted: 25 February 2019

Published online: 21 March 2019

\section{References}

1. Huang, Y., Li, W. \& Yan, D. Preparation and characterization of a series of polyamides with long alkylene segments: nylons 12 20, 10 20, S 20, 620,420 and 2 20. Polym. Bull. 49, 111-118 (2002).

2. Ehrenstein, M., Smith, P. \& Weder, C. Polyamides X. 34: A new class of polyamides with long alkane segments. Macromol. Chem. Phys. 204, 1599-1606 (2003).

3. Liu, C. et al. Polymers from fatty acids: poly ( $\Omega$-hydroxyl tetradecanoic acid) synthesis and physico-mechanical studies. Biomacromolecules 12, 3291-3298 (2011).

4. De Ten Hove, C. L. F., Penelle, J., Ivanov, D. A. \& Jonas, A. M. Encoding crystal microstructure and chain folding in the chemical structure of synthetic polymers. Nat. Mater. 3, 33 (2004).

5. van der Meulen, I. et al. Polymers from functional macrolactones as potential biomaterials: enzymatic ring opening polymerization, biodegradation, and biocompatibility. Biomacromolecules 9, 3404-3410 (2008).

6. Quinzler, D. \& Mecking, S. Linear Semicrystalline polyesters from fatty acids by complete feedstock molecule utilization. Angew. Chem. Int. Ed. 49, 4306-4308 (2010).
7. Saini, P. K., Fiorani, G., Mathers, R. T. \& Williams, C. K. Zinc versus magnesium: orthogonal catalyst reactivity in selective polymerizations of epoxides, bio-derived anhydrides and carbon dioxide. Chem. Eur. J. 23, 4260-4265 (2017).

8. Zhu, Y., Romain, C. \& Williams, C. K. Sustainable polymers from renewable resources. Nature 540, 354-362 (2016).

9. Hillmyer, M. A. \& Tolman, W. B. Aliphatic polyester block polymers: renewable, degradable, and sustainable. Acc. Chem. Res. 47, 2390-2396 (2014).

10. Schneiderman, D. K. \& Hillmyer, M. A. 50th Anniversary perspective: there is a great future in sustainable polymers. Macromolecules 50, 3733-3749 (2017).

11. Wang, Z., Yuan, L. \& Tang, C. Sustainable elastomers from renewable biomass. Acc. Chem. Res. 50, 1762-1773 (2017).

12. Yao, K. \& Tang, C. Controlled polymerization of next-generation renewable monomers and beyond. Macromolecules 46, 1689-1712 (2013).

13. Hong, M. \& Chen, E. Y. X. Chemically recyclable polymers: a circular economy approach to sustainability. Green Chem. 19, 3692-3706 (2017).

14. Kristufek, S. L., Wacker, K. T., Tsao, Y.-Y. T., Su, L. \& Wooley, K. L. Monomer design strategies to create natural product-based polymer materials. Nat. Prod. Rep. 34, 433-459 (2017).

15. Gandini, A. \& Lacerda, T. M. From monomers to polymers from renewable resources: recent advances. Prog. Polym. Sci. 48, 1-39 (2015).

16. Garcia, J. M. \& Robertson, M. L. The future of plastics recycling. Science 358 870 (2017).

17. Miller, S. A. Sustainable polymers: opportunities for the next decade. ACS Macro Lett. 2, 550-554 (2013). 
18. Stempfle, F., Quinzler, D., Heckler, I. \& Mecking, S. Long-chain linear C19 and C23 monomers and polycondensates from unsaturated fatty acid esters. Macromolecules 44, 4159-4166 (2011).

19. Stempfle, F., Ortmann, P. \& Mecking, S. Long-chain aliphatic polymers to bridge the gap between semicrystalline polyolefins and traditional polycondensates. Chem. Rev. 116, 4597-4641 (2016).

20. Corma, A., Iborra, S. \& Velty, A. Chemical routes for the transformation of biomass into chemicals. Chem. Rev. 107, 2411-2502 (2007).

21. Shao, Z. \& Vollrath, F. Surprising strength of silkworm silk. Nature 418, 741 (2002).

22. Simmons, A. H., Michal, C. A. \& Jelinski, L. W. Molecular orientation and two-component nature of the crystalline fraction of spider dragline silk. Science 271, 84-87 (1996).

23. Lefèvre, T., Rousseau, M. E. \& Pézolet, M. Protein secondary structure and orientation in silk as revealed by raman spectromicroscopy. Biophys. J. 92, 2885 (2007).

24. Hong, Y., Lam, J. W. \& Tang, B. Z. Aggregation-induced emission: phenomenon, mechanism and applications. Chem. Commun. 40, 4332-4353 (2009)

25. Luo, J. et al. Aggregation-induced emission of 1-methyl-1,2,3,4,5pentaphenylsilole. Chem. Commun. 0, 1740-1741 (2001).

26. Mei, J., Leung, N. L., Kwok, R. T., Lam, J. W. \& Tang, B. Z. Aggregationinduced emission: together we shine, united we soar! Chem. Rev. 115, 11718-11940 (2015).

27. Zhou, Q. et al. Clustering-triggered emission of nonconjugated polyacrylonitrile. Small 12, 6586-6592 (2016).

28. Zhao, E. et al. Poly[(Maleic Anhydride)-Alt-(Vinyl Acetate)]: a pure oxygenic nonconjugated macromolecule with strong light emission and solvatochromic effect. Macromolecules 48, 64-71 (2014).

29. $\mathrm{Lu}, \mathrm{H}$. et al. Unexpected strong blue photoluminescence produced from the aggregation of unconventional chromophores in novel siloxane-poly (amidoamine) dendrimers. Macromolecules 48, 476-482 (2015).

30. Kakuchi, R. Multicomponent reactions in polymer synthesis. Angew. Chem. Int. Ed. 53, 46 (2014).

31. Sehlinger, A., Dannecker, P. K., Kreye, O. \& Meier, M. A. R. Diversely substituted polyamides: macromolecular design using the Ugi fourcomponent reaction. Macromolecules 47, 2774-2783 (2014).

32. Wang, Y. Z. et al. One-pot synthesis of polyamides with various functional side groups via Passerini reaction. Polym. Chem. 4, 444-448 (2013).

33. Turunc, O., Firdaus, M., Klein, G. \& Meier, M. A. R. Fatty acid derived renewable polyamides via thiol-ene additions. Green Chem. 14, 2577-2583 (2012).

34. Song, L., Wang, Z., Lamm, M. E., Yuan, L. \& Tang, C. Supramolecular polymer nanocomposites derived from plant oils and cellulose nanocrystals. Macromolecules 50, 7475-7483 (2017).

35. Yuan, L., Wang, Z., Trenor, N. M. \& Tang, C. Robust amidation transformation of plant oils into fatty derivatives for sustainable monomers and polymers. Macromolecules 48, 1320-1328 (2015)

36. Yuan, L., Wang, Z., Trenor, N. M. \& Tang, C. Amidation of triglycerides by amino alcohols and their impact on plant oil-derived polymers. Polym. Chem. 7, 2790-2798 (2016).

37. Li, L., Koch, M. H. \& de Jeu, W. H. Crystalline structure and morphology in Nylon-12: a small-and wide-angle X-ray scattering study. Macromolecules 36, 1626-1632 (2003).

38. Ramesh, C. Crystalline transitions in Nylon 12. Macromolecules 32, 5704-5706 (1999).

39. Dencheva, N., Nunes, T. G., Oliveira, M. J. \& Denchev, Z. Crystalline structure of polyamide 12 as revealed by solid-state ${ }^{13} \mathrm{C}$ NMR and synchrotron Waxs and Saxs. J. Polym. Sci. Part B 43, 3720-3733 (2005).

40. Waletzko, R. S., Korley, L. S. T. J., Pate, B. D., Thomas, E. L. \& Hammond, P. T. Role of increased crystallinity in deformation-induced structure of segmented thermoplastic polyurethane elastomers with Peo and Peo-Ppo -Peo soft segments and Hdi. Macromolecules 42, 2041-2053 (2009).

41. Zhang, K. et al. Non-isocyanate poly (amide-hydroxyurethane) S from sustainable resources. Green Chem. 18, 4667-4681 (2016).

42. Degtyar, E., Harrington, M. J., Politi, Y. \& Fratzl, P. The mechanical role of metal ions in biogenic protein-based materials. Angew. Chem. Int. Ed. 53, 12026-12044 (2014)

43. Tang, Z., Huang, J., Guo, B., Zhang, L. \& Liu, F. Bioinspired engineering of sacrificial metal-ligand bonds into elastomers with supramechanical performance and adaptive recovery. Macromolecules 49, 1781-1789 (2016)

44. Chen, Z. \& Lu, H. Constructing sacrificial bonds and hidden lengths for ductile graphene/polyurethane elastomers with improved strength and toughness. J. Mater. Chem. 22, 12479 (2012).

45. Wang, Z. et al. Bioinspired design of nanostructured elastomers with crosslinked soft matrix grafting on the oriented rigid nanofibers to mimic mechanical properties of human skin. ACS Nano 9, 271-278 (2015).

46. Wang, Z. et al. Bioinspired high resilient elastomers to mimic resilin. ACS Macro Lett. 5, 220-223 (2016).
47. Mullins, L. Softening of rubber by deformation. Rubber Chem. Technol. $\mathbf{4 2}$ 339-362 (2012)

48. Davis, A. V. \& O'Halloran, T. V. A place for thioether chemistry in cellular copper ion recognition and trafficking. Nat. Chem. Biol. 4, 148 (2008).

49. Knorr, $\mathrm{M}$. et al. Construction of (Cux)2n cluster-containing ( $\mathrm{X}=\mathrm{Br}, \mathrm{I} ; \mathrm{N}=1,2)$ coordination polymers assembled by dithioethers $\mathrm{Ars}(\mathrm{Ch} 2) \mathrm{Msar}(\mathrm{Ar}=\mathrm{Ph}, \mathrm{P}-$ Tol; $M=3,5$ ): effect of the spacer length, aryl group, and metal-to-ligand ratio on the dimensionality, cluster nuclearity, and the luminescence properties of the metal-organic frameworks. Inorg. Chem. 51, 9917-9934 (2012).

50. Lapprand, A. et al. Formation of an unprecedented (Cubr) 5 cluster and a zeolitetype $2 \mathrm{~d}$-coordination polymer: a surprising halide effect. Chem. Commun. 49, 8848-8850 (2013)

51. Zhang, J., Li, M., Cheng, L. \& Li, T. Multifunctional polymers built on copper-thioether coordination. Polym. Chem. 8, 6527-6533 (2017).

52. Hong, Y., Lam, J. W. \& Tang, B. Z. Aggregation-induced emission. Chem. Soc Rev. 40, 5361 (2011).

53. Yuan, W. Z. et al. Crystallization-induced phosphorescence of pure organic luminogens at room temperature. J. Phys. Chem. C 114, 6090-6099 (2010).

54. Lin, Y., Gao, J. W., Liu, H. W. \& Li, Y. S. Synthesis and characterization of hyperbranched poly(ether amide)S with thermoresponsive property and unexpected strong blue photoluminescence. Macromolecules 42, 3237-3246 (2009).

55. Mohamed, M. G., Hsu, K.-C., Hong, J.-L. \& Kuo, S.-W. Unexpected fluorescence from maleimide-containing polyhedral oligomeric silsesquioxanes: nanoparticle and sequence distribution analyses of polystyrene-based alternating copolymers. Polym. Chem. 7, 135-145 (2016).

56. Tomalia, D. A. et al. Non-traditional intrinsic luminescence: inexplicable blue fluorescence observed for dendrimers, macromolecules and small molecular structures lacking traditional/conventional luminophores. Prog. Polym. Sci. 90, 35-117 (2019).

\section{Acknowledgements}

Z.W. acknowledges National Natural Science Foundation of China for financial support (grant nos. 51773001, 51603002), and the financial support from Natural Science Foundation for Distinguished Young Scholar of Anhui Province (grant no. 1808085J26) C.T. would like to acknowledge the support from the US National Science Foundation (DMR-1806792). This work made use of the South Carolina SAXS Collaborative, supported by the NSF Major Research Instrumentation program (award no. DMR-1428620). We thank Prof. Ben Zhong Tang for constructive discussions on the AIE effect.

\section{Author contributions}

Z.W. and C.T. designed the study. L.S. and T.Z. contributed equally to this work and carried out most of the experiments; L.Y., J.Z. and Y.Z. participated in the experiments for mechanical and thermal property measurements and the related data analysis. All authors participated in the interpretation of the data and production of the final manuscript.

\section{Additional information}

Supplementary Information accompanies this paper at https://doi.org/10.1038/s41467 019-09218-6.

Competing interests: The authors declare no competing interests.

Reprints and permission information is available online at http://npg.nature.com/ reprintsandpermissions/

Journal peer review information: Nature Communications thanks Jinwen Zhang and the other anonymous reviewer(s) for their contribution to the peer review of this work

Publisher's note: Springer Nature remains neutral with regard to jurisdictional claims in published maps and institutional affiliations.

Open Access This article is licensed under a Creative Commons Attribution 4.0 International License, which permits use, sharing, adaptation, distribution and reproduction in any medium or format, as long as you give appropriate credit to the original author(s) and the source, provide a link to the Creative Commons license, and indicate if changes were made. The images or other third party material in this article are included in the article's Creative Commons license, unless indicated otherwise in a credit line to the material. If material is not included in the article's Creative Commons license and your intended use is not permitted by statutory regulation or exceeds the permitted use, you will need to obtain permission directly from the copyright holder. To view a copy of this license, visit http://creativecommons.org/ licenses/by/4.0/

(c) The Author(s) 2019 